\title{
Optical and Electrical Properties of Chemical Bath Deposited Cobalt Sulphide Thin Films
}

\author{
Geetha Govindasamy ${ }^{a}$, Priya Murugasen ${ }^{b *}$, Suresh Sagadevan ${ }^{c}$ \\ ${ }^{a}$ R\&D Centre, Bharathiar University, Coimbatore, India \\ ${ }^{b}$ Department of Physics, Saveetha Engineering, Chennai, Tamil Nadu, India \\ ${ }^{c}$ Department of Physics, AMET University, Chennai, Tamil Nadu, India
}

Received: June 10, 2016; Revised: August 14, 2016; Accepted: September 7, 2016

\begin{abstract}
Cobalt sulphide (CoS) thin films were synthesized using the Chemical Bath Deposition (CBD) technique. X-ray diffraction (XRD) analysis was used to study the structure and the crystallite size of CoS thin film. Scanning Electron Microscope (SEM) studies reveal the surface morphology of these films. The optical properties of the CoS thin films were determined using UV-Visible absorption spectrum. The optical band gap of the thin films was found to be $1.6 \mathrm{eV}$. Optical constants such as the refractive index, the extinction coefficient and the electric susceptibility were determined. The dielectric studies were carried out at different frequencies and at different temperatures for the prepared $\mathrm{CoS}$ thin films. In addition, the plasma energy of the valence electron, Penn gap or average energy gap, the Fermi energy and electronic polarizability of the thin films were determined. The AC electrical conductivity measurement was also carried out for the thin films. The activation energy was determined by using DC electrical conductivity measurement.
\end{abstract}

Keywords: $C O S, C B D, X R D$, SEM, Dielectric studies, $A C$ and DC electrical conductivity measurement

\section{Introduction}

Thin films of metal chalcogenide have been studied extensively in recent years due to their applications in photovoltaic and opto-electronic devices ${ }^{1}$. Thin films are thin material layers ranging from fractions of a nanometer to several micrometers in thickness. Cobalt sulphide (CoS) has wide range of applications in solar selective coatings, IR detectors and as a storage electrode in photoelectrochemical storage device. Growth of the metal chalcogenide thin films via chemical solution deposition or chemical bath deposition or chemical growth process route is one of the key technologies today ${ }^{2-4}$. The $\operatorname{CoS}$ thin films can be prepared using chemical bath deposition (CDB) method ${ }^{5,6}$. Recently, synthesis of CoS thin films was carried out by a modified liquid phase chemical growth process $^{7,8}$. In chemical methods, the deposition parameters such as $\mathrm{pH}$, temperature, concentration of the solution and deposition time influence the formation of nanocrystalline thin films and crystallite size, which in turn engineer the bandgap of the material. Chemical bath deposition technique (CBD) is one of the most promising methods for making large area high quality nanocrystalline thin films for photovoltaic applications because it is an efficient and cost effective method. In this study, chemical bath deposition technique was employed to deposit the thin film of cobalt sulphide on microscopic glass. The prepared films were characterized for their structural, surface morphology, optical, and electrical properties.

* e-mail: priyamurugasen15@gmail.com

\section{Experimental Procedure}

Chemical bath deposition technique was used for the deposition of CoS thin films. The substrates were washed using soap solution and subsequently kept in hot chromic acid and then ultrasonically cleaned with deionized water for $10 \mathrm{~min}$ and wiped with acetone and stored in a hot oven. The growth solution consisted of $10 \mathrm{ml}$ of Cobalt (II) sulfate, $2 \mathrm{ml}$ triethanolamine, $15 \mathrm{ml}$ ammonia, $10 \mathrm{ml}$ of thiourea and double distilled water, which were mixed thoroughly. The deposition was carried out at a temperature of $110^{\circ} \mathrm{C}$. A glass substrate was placed vertically inside the vessel with the help of a suitably designed substrate holder. After a time period of $60 \mathrm{~min}$, the glass slide was removed from the bath and cleaned with deionized water and dried in the hot oven. Many trials were made by optimizing the deposition parameters to obtain a good quality $\operatorname{CoS}$ thin film. The resultant films were found to be homogeneous and well adhered to the substrate with mirror like surface. The XRD pattern of the CoS thin films was recorded by using powder X-ray diffractometer (Schimadzu model: XRD 6000 and $\mathrm{CuK} \alpha(\lambda=0.154 \mathrm{~nm})$ ) with diffraction angle between $20^{\circ}$ and $70^{\circ}$. The crystallite size was determined using the Debye Scherrer's formula for the broadenings of the corresponding X-ray spectral peaks. A VARIAN CARY MODEL 5000 spectrophotometer was used for recording the optical absorption spectrum in the wavelength range of $400-800 \mathrm{~nm}$. The dielectric properties of the CoS thin films were analyzed using a HIOKI 3532-50 LCR HITESTER for a frequency range from $50 \mathrm{~Hz}-5 \mathrm{MHz}$. 


\section{Results and Discussion}

\subsection{Structural characterization}

The XRD pattern of the CoS thin films was recorded by using a powder X-ray diffractometer, with a diffraction angle between $20^{\circ}$ and $70^{\circ}$ and is shown in Figure 1. The pattern revealed hexagonal wurtzite polycrystalline structure of CoS films. The well defined peaks were observed in the pattern. The excellent peaks (100), (101), (102), (110), (103), (200), and (112) were obtained in the powder X-ray diffraction studies. No impurity peaks were observed in the XRD patterns. The average crystalline size (D) was calculated using the Debye-Scherrer formula,

$$
D=\frac{0.9 \lambda}{\beta \cos \theta}
$$

where $\lambda$ is the $\mathrm{X}$-ray wavelength, $\theta$ is the Bragg diffraction angle, and $\beta$ is the FWHM of the XRD peak appearing at the diffraction angle $\theta$. The average crystalline size was calculated from X-ray line broadening peak and DebyeScherrer equation and it was found to be about $14 \mathrm{~nm}$.

\subsection{Surface Analysis}

Scanning Electron Microscope (SEM) is very helpful to study the surface morphology of films. SEM images give information regarding the surface structure and roughness. SEM image obtained for $\mathrm{CoS}$ thin film deposited on glass substrate is shown in Figure 2. The CoS thin films exhibited more or less uniform surface morphology. The thickness of the obtained film is $2 \mathrm{~mm}$ and the mean crystallite size was found to be about12 $\mathrm{nm}$.

\subsection{UV-Visible spectroscopy-}

Optical investigations of the prepared films revealed that there was a band to band direct transition. The optical absorption spectrum of the CoS thin film was obtained in the wavelength range of $400-800 \mathrm{~nm}$. Figure 3 shows the variation of the optical absorbance with the wavelength of the CoS thin films which shows the edge shift towards longer wavelength region (red shift). It is observed that the optical absorption decreases smoothly from UV to near IR region. This validates the material's suitability in devices used for good absorption of UV radiation, that is to say, it can be used as a UV filter ${ }^{9}$. The optical absorption coefficient $(\alpha)$ was calculated from transmittance using the relation

$$
\alpha=\frac{1}{d} \log \left(\frac{1}{T}\right)
$$

where $\mathrm{T}$ is the transmittance and $\mathrm{d}$ is the thickness of the films. The films under study have an absorption coefficient $(\alpha)$ obeying the following relation for high photon energies $(\mathrm{h} v)$

$$
\alpha=\frac{A\left(h v-E_{g}\right)^{1 / 2}}{h v}
$$

where $\mathrm{E}_{\mathrm{g}}$ is the optical band gap of the films and $\mathrm{A}$ is a constant. The fundamental absorption corresponding to the optical transition of the electrons from the valence band to the conduction band can be used to determine the nature and value of the optical band gap $E g$ of the films. A plot of variation of $(\alpha h v)^{2}$ versus $h v$ is shown in Figure 4 . $\mathrm{E}_{\mathrm{g}}$ could be evaluated using the extrapolation of the linear part. The intercept on energy axis gives the value of band gap energy and it was found to be $1.6 \mathrm{eV}$. This bandgap narrowing indicates that the top of the valence band and the bottom of the conduction band are modified with increasing substrate temperature to various extents ${ }^{10,11}$.

\subsubsection{Determination of Optical Constants}

Two of the most important optical properties, viz., the refractive index (n) and the extinction coefficient $(\mathrm{K})$ are generally called optical constants. The amount of light that transmits through thin film material depends on the amount of the reflection and the absorption that take place along the light path. The optical constants such as the refractive index (n), the real dielectric constant $\left(\mathrm{e}_{\mathrm{r}}\right)$ and the imaginary part of the dielectric constant $\left(\mathrm{e}_{\mathrm{i}}\right)$ were calculated. The extinction coefficient $(\mathrm{K})$ was obtained from the following equation

$$
K=\frac{\lambda \alpha}{4 \pi}
$$

The extinction coefficient $(\mathrm{K})$ was found to be 0.0044 at $\lambda=800 \mathrm{~nm}$. The transmittance $(\mathrm{T})$ is given by

$$
T=\frac{(1-R)^{2} \exp (-\alpha t)}{1-R^{2} \exp (-2 \alpha t)}
$$

Reflectance (R) in terms of absorption coefficient was obtained from the above equation.

Hence,

$$
R=\frac{1 \pm \sqrt{1-\exp (-\alpha t+\exp (\alpha t))}}{1+\exp (-\alpha t)}
$$

Refractive index (n) could be determined from the reflectance data using the following equation

$$
n=\frac{(R+1) \pm \sqrt{3 R^{2}+10 R-3}}{2(R-1)}
$$


The refractive index (n) was found to be 1.43 at $\lambda=800$ $\mathrm{nm}$. From the optical constants, electric susceptibility $\left(\chi_{\mathrm{c}}\right)$ could be calculated from the following relation

$$
\varepsilon_{r}=\varepsilon_{0}+4 \pi \chi_{C}=n^{2}-k^{2}
$$

Hence, we have

$$
\chi_{C}=\frac{n^{2}-k^{2}-\varepsilon_{0}}{4 \pi}
$$

Where $\varepsilon_{0}$ is the permittivity of free space. The value of the electric susceptibility $\left(\chi_{\mathrm{c}}\right)$ was 1.044 at $\lambda=800 \mathrm{~nm}$. Since electrical susceptibility was greater than 1 , the material could be easily polarized when the incident light was more intense. The real part of the dielectric constant $\left(\varepsilon_{\mathrm{r}}\right)$ and the imaginary part of the dielectric constant $\left(\varepsilon_{\mathrm{i}}\right)$ could be calculated from the following relations

$$
\begin{gathered}
\varepsilon_{r}=n^{2}-k^{2} \\
\varepsilon_{i}=2 n k
\end{gathered}
$$

The values of the real dielectric constant $\left(\varepsilon_{\mathrm{r}}\right)$ and the imaginary dielectric constant $\left(\varepsilon_{\mathrm{i}}\right)$ at $\lambda=800 \mathrm{~nm}$ were estimated to be 3.241 and 0.0041 respectively. The lower value of the dielectric constant made it possible to cause induced polarization due to intense incident light radiation.

\subsection{Dielectric Studies}

The electrical properties of the materials are of great importance in determining the usage of the film for photovoltaic applications. The electrical property depends on various growth parameters such as film composition, thickness, rate of deposition, and substrate temperature. The dielectric constant and the dielectric loss were calculated at

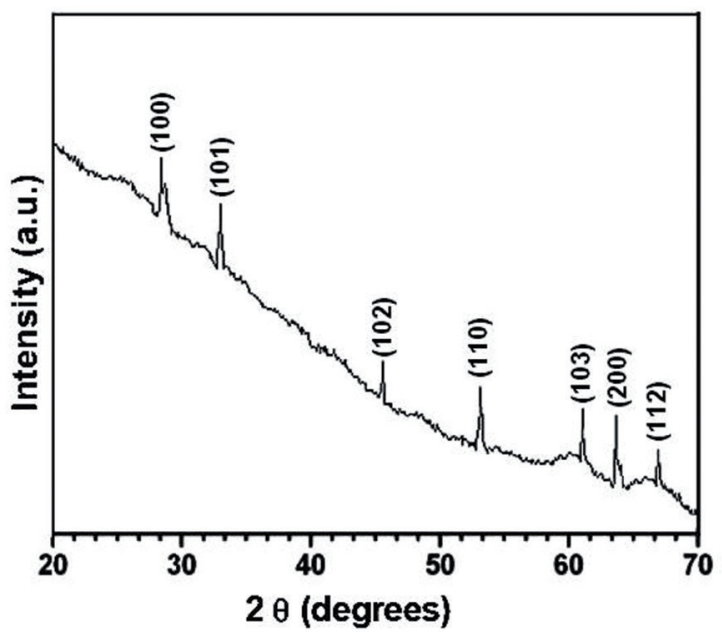

Figure 1: XRD spectrum of $\mathrm{CoS}$ thin films.

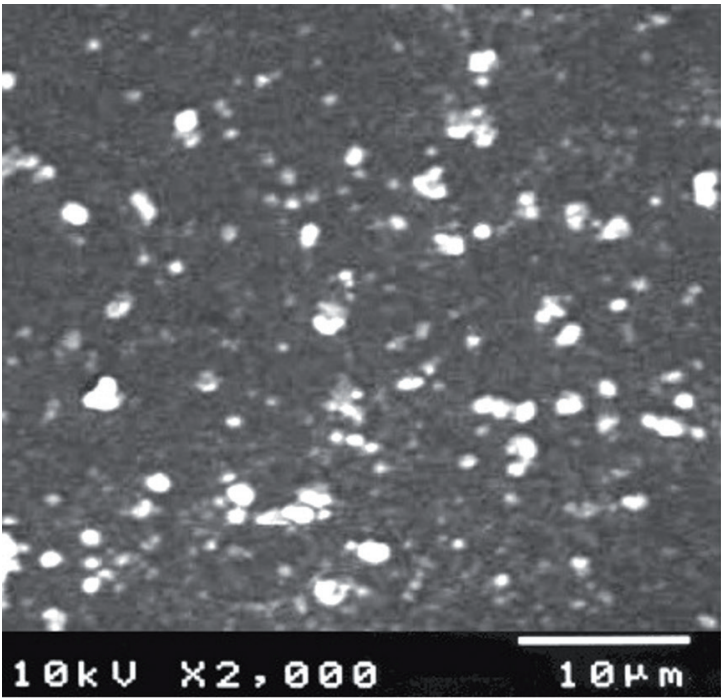

Figure 2: SEM Image of the CoS thin films

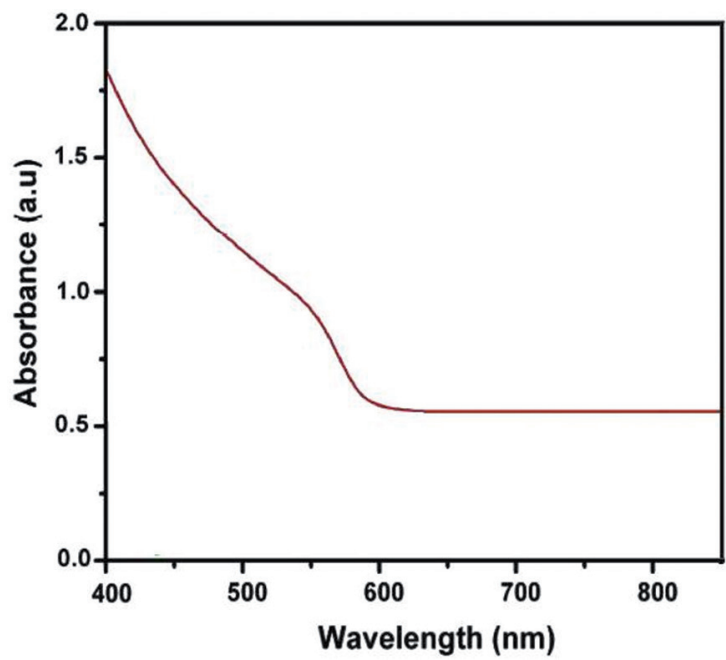

Figure 3: Optical absorption spectrum of CoS thin film

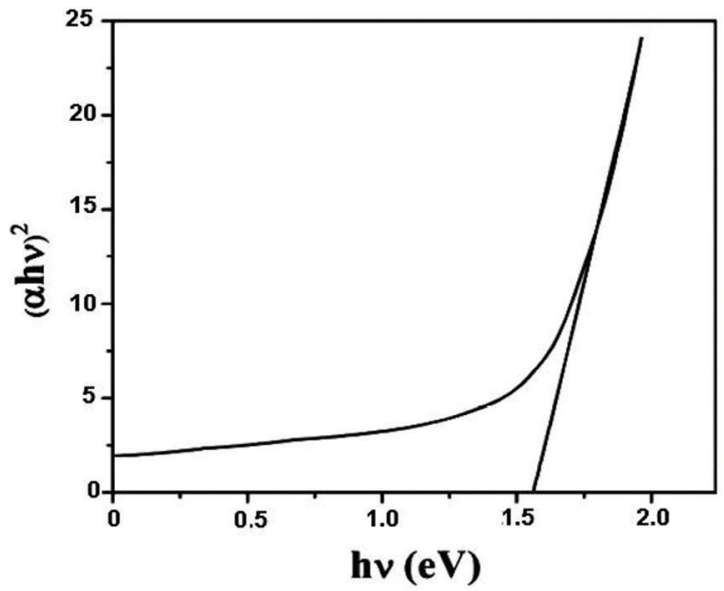

Figure 4: Plot of $(\alpha h v)^{2}$ versus hv 
different frequencies and temperatures and their variations with $\log f$ are shown in Figures 5 and 6. It is observed that the dielectric constant decreases with rising frequency and then achieves almost a constant value in the high frequency region. The net polarization of $\mathrm{CoS}$ thin film is owing to ionic, electronic, dipolar and space charge polarizations. The huge valued dielectric constant at low frequencies can be attributed to the lower electrostatic binding strength, arising due to the space charge polarization near the grain boundary interfaces ${ }^{12}$. Owing to the application of an electric field, the space charges are stimulated and dipole moments are produced and this is called space-charge polarization ${ }^{13}$. Apart from this, these dipole moments are rotated by the field applied ensuing in rotation polarization which also contributes to the high values. Figure 6 shows the dielectric loss as a function of frequency. These curves show that comparable to that of the dielectric constant, the dielectric loss depends on the frequency of the applied field. The dielectric loss decreases with an increase in the frequency at almost all temperatures, but appears to attain saturation in the higher frequency range at all the temperatures ${ }^{14}$. The amount of charge carriers increases by thermal activation and the place of loss peak shifts to top frequency with increasing temperature being indicated by the rise in the maximum out value of dielectric loss with temperature ${ }^{15}$.

The high frequency dielectric constant is required as input, to evaluate electronic properties such as valence electron plasma energy, average energy gap or Penn gap, Fermi energy and electronic polarizability of the CoS thin films. The theoretical calculations showed that the high frequency dielectric constant was explicitly dependent on the valence electron Plasma energy, an average energy gap referred to as the Penn gap and Fermi energy. The Penn gap is determined by fitting the dielectric constant with the Plasmon energy.

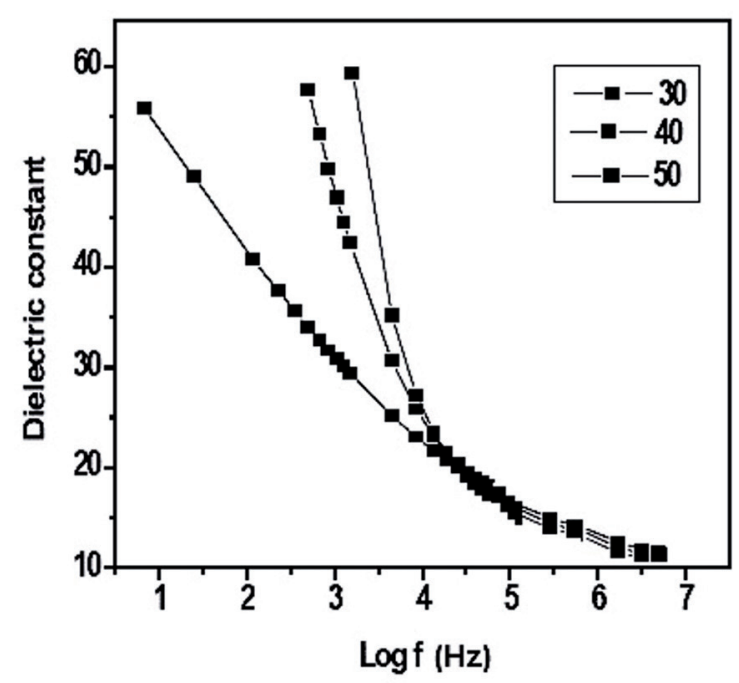

Figure 5: Dielectric constant of $\mathrm{CoS}$ thin film

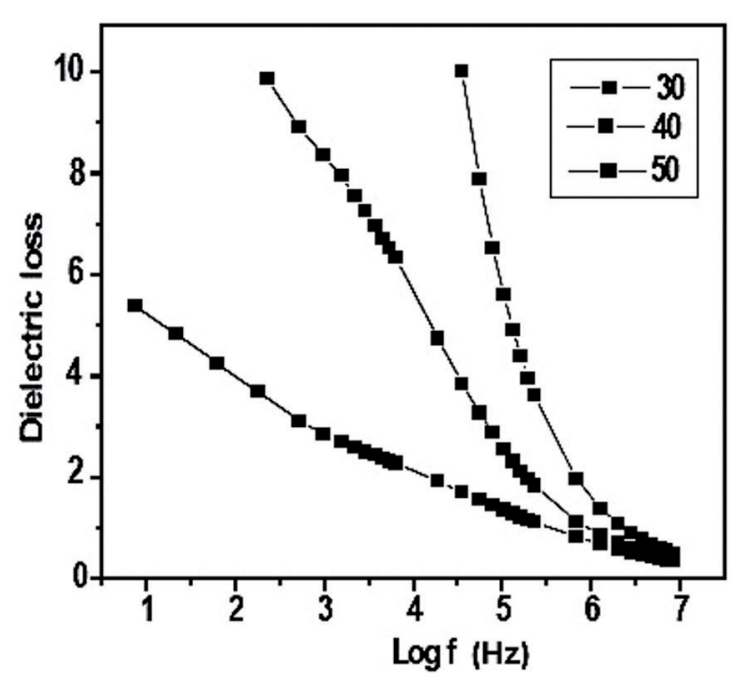

Figure 6: Dielectric loss of CoS thin film

The following relation was used to calculate the valence electron plasma energy, $\hbar \omega_{\mathrm{p}}$

$$
\hbar \omega_{P}=28.8\left(\frac{Z \rho}{M}\right)^{1 / 2}
$$

According to the Penn model, the average energy gap for the $\mathrm{CoS}$ thin films is given by

$$
E_{P}=\frac{\hbar \omega p}{\left(\varepsilon_{\infty}-1\right)^{1 / 2}}
$$

Where $\hbar \omega_{P}$ is the valence electron plasmon energy and the Fermi energy is given by

$$
E_{F}=0.2948\left(\hbar \omega_{P}\right)^{4 / 3}
$$

Then, the electronic polarizability ( $\alpha$ ), is given by

$$
\begin{aligned}
& \alpha=\left[\frac{\left(\hbar \omega_{P}\right)^{2} S_{0}}{\left(\hbar \omega_{P}\right)^{2} S_{0}+3 E_{P}^{2}}\right] \times \\
& \frac{M}{\rho} \times 0.396 \times 10^{-24} \mathrm{~cm}^{3}
\end{aligned}
$$

Where $S_{0}$ is a constant given by

$$
S_{0}=1-\left[\frac{E_{P}}{4 E_{F}}\right]+\frac{1}{3}\left[\frac{E_{P}}{4 E_{F}}\right]^{2}
$$

The Clausius-Mossotti relation also gives $\alpha$ :

$$
\alpha=\frac{3}{4} \frac{M}{\pi N \alpha \rho}\left[\frac{\varepsilon_{\infty}-1}{\varepsilon_{\infty}+2}\right]
$$

The following empirical relationship is also used to calculate $\alpha$ : 
$\alpha=\left[-1 \frac{\sqrt{E_{g}}}{4.06}\right] \times \frac{M}{\rho} \times 0.396 \times 10^{-24} \mathrm{~cm}^{3}$

where $\mathrm{E}_{\mathrm{g}}$ is the bandgap value determined through the UV-Visible spectrum. The high frequency dielectric constant of the materials is a very important parameter for calculating the physical or electronic properties of materials ${ }^{16}$. All the above parameters as estimated from our experiments are shown in Table 1.

Table 1: Electronic parameters of the CoS thin films

\begin{tabular}{lc}
\hline Parameter & Value \\
\hline Plasma energy $\left(\mathrm{h} \omega_{\mathrm{p}}\right)$ & $19.93 \mathrm{eV}$ \\
Penn gap $\left(\mathrm{E}_{\mathrm{p}}\right)$ & $1.55 \mathrm{eV}$ \\
Fermi Energy $\left(\mathrm{E}_{\mathrm{F}}\right)$ & $15.77 \mathrm{eV}$ \\
$\begin{array}{l}\text { Electronic polarizability (using the Penn } \\
\text { analysis) }\end{array}$ & $4.72 \times 10^{-24} \mathrm{~cm}^{3}$ \\
$\begin{array}{l}\text { Electronic polarizability (using the Clausius- } \\
\text { Mossotti relation) }\end{array}$ & $4.84 \times 10^{-24} \mathrm{~cm}^{3}$ \\
Electronic polarizability (using bandgap) & $4.55 \times 10^{-24} \mathrm{~cm}^{3}$ \\
\hline
\end{tabular}

\subsection{AC conductivity $\left(\sigma_{\mathrm{ac}}\right)$}

AC conductivity measurements were made in the frequency range $20 \mathrm{~Hz}$ to $1 \mathrm{MHz}$ using HIOKI 3532-50 LCR HITESTER. A chromel-Alumel thermocouple was employed to record the sample temperature. A 30 minute interval was maintained prior to thermal stabilization after each measuring temperature. The ac conductivity $\left(\sigma_{a c}\right)$ was calculated using the formula

$$
\sigma_{a c}=\varepsilon_{0} \varepsilon_{r} \omega \tan \delta
$$

Where $\varepsilon_{\mathrm{o}}$ is the vacuum dielectric constant $\left(8.85 \times 10^{-12}\right.$ farad $/ \mathrm{m}), \varepsilon_{\mathrm{r}}$ is the relative dielectric constant and $\omega$ is the angular frequency $\omega=2 \pi v$ of the applied field. Figure 7 shows the variation of ac conductivity with frequency and temperature. It is seen that the value of ac conductivity increases with increase in the frequency. There is a small increase in the electrical conductivity of the nanomaterial for an increase in frequency and is the same for all temperatures at the low frequency region. The increase in conductivity with temperature may be explained based on the assumption that within the bulk, the oxygen vacancies due to the loss of oxygen are usually created during increase in temperature and the charge compensation, which would leave behind free electrons at higher frequencies only. In metal oxides, electrical conduction occurs through strong coupling between phonons and electrons with the creation of polarons.

\subsection{DC conductivity $\left(\sigma_{d e}\right)$}

The de conductivity measurements of $\mathrm{CoS}$ thin films were carried out. The de conductivity $\sigma_{d c}$ of the $\operatorname{CoS}$ thin films was calculated using the relation

$$
\sigma_{d c}=\frac{t}{R A}
$$

Where, $\mathrm{R}$ is the measured resistance, $\mathrm{t}$ is the thickness of the films and $\mathrm{A}$ is the area of the films in contact with the electrode. Figure 8 shows the variation of $\sigma_{\mathrm{dc}}$ conductivity versus temperatures. It could be seen that the dc conductivity was increasing with increase in temperature ${ }^{17}$. Traps which are present in the CoS thin films may be filled by excitation at low temperature and may be emptied by raising the temperature by thermal activation. Upon thermal activation, the mobility of charge carriers increases with a possible mechanism of hopping to cross the potential barrier and hence produce enhanced current. The value of activation energy was determined from the slope of $\ln \sigma_{\mathrm{dc}}$ versus $1000 / \mathrm{T}$ (Figure 9) which was found to be $0.40 \mathrm{eV}$.

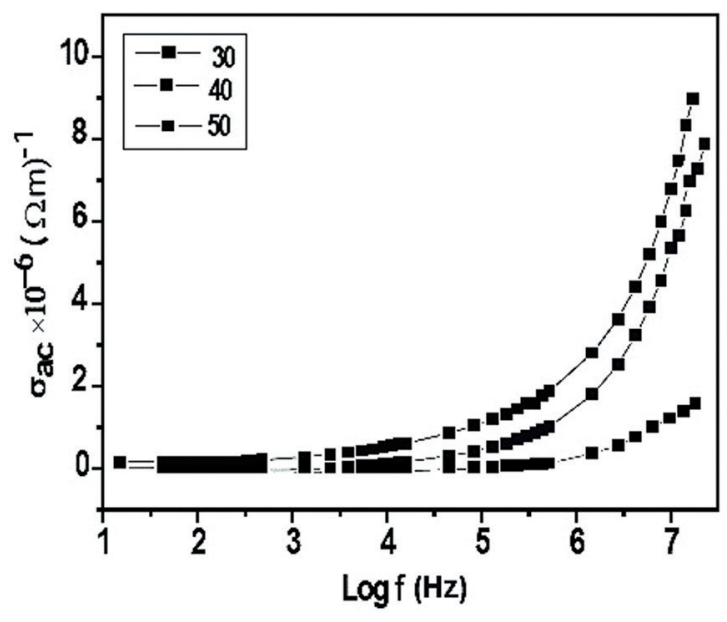

Figure 7: Variation of ac conductivity with frequency at various temperatures.

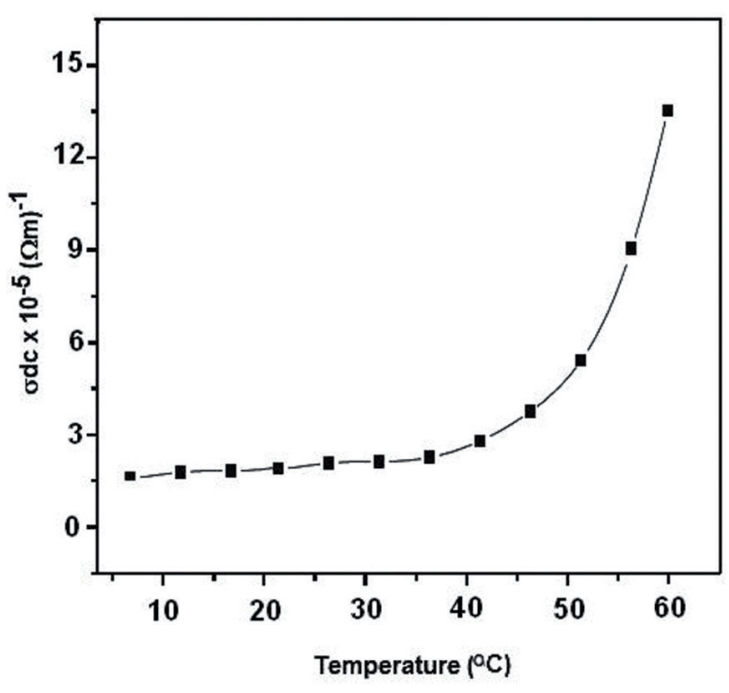

Figure 8: Variation of dc conductivity versus temperature. 


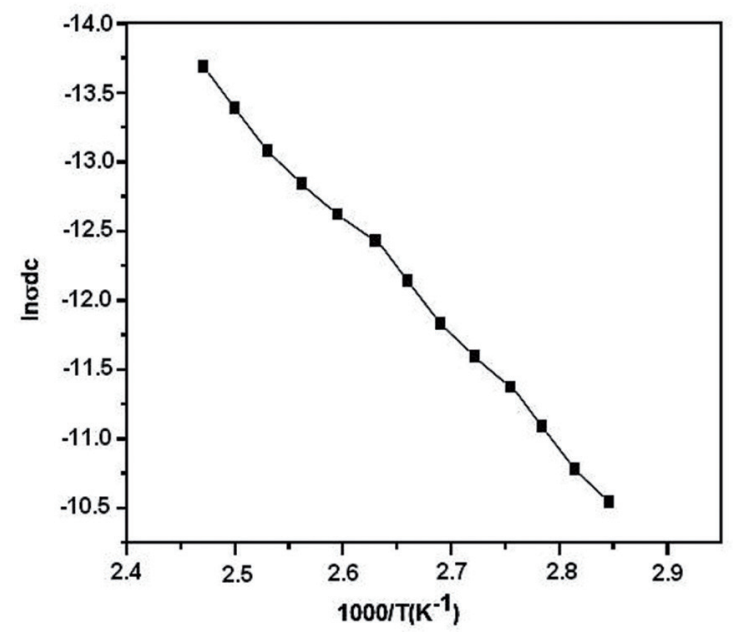

Figure 9: Plot of $\ln \sigma_{\mathrm{dc}}$ versus $1000 / \mathrm{T}\left(\mathrm{K}^{-1}\right)$

\section{Conclusions}

The $\mathrm{CoS}$ thin film was synthesized by $\mathrm{CBD}$ technique. The XRD studies revealed that the $\mathrm{CoS}$ thin film was in hexagonal phase and the crystallite size was found to be 14 $\mathrm{nm}$. The morphology of the CoS thin films was characterized by using SEM. The optical band gap was found to be 1.6 $\mathrm{eV}$. The optical constants such as the refractive index, the extinction coefficient and the electrical susceptibility were calculated. The dielectric properties of the CoS thin films were calculated at different frequencies and temperatures. In addition, the plasma energy of the valence electron, Penn gap or average energy gap, the Fermi energy, and electronic polarizability of CoS thin films were also determined. The $\mathrm{AC}$ electrical conductivity was found to increase with an increase in the temperature and frequency. The DC conductivity study also revealed that the DC conductivity increased with increase in temperature. The activation energy was found to be $0.40 \mathrm{eV}$. The obtained values for electrical conductivity and optical constants made the material to be a good candidate for photovoltaic and opto-electronic applications.

\section{References}

1. Cruz-Vázquez C, Inoue M, Inoue MB, Bernal R, Espinza Beltrán FJ. Electrical and spectroscopic properties of amorphous copper sulfide films treated with iodine, lithium iodide and sodium iodide. Thin Solid Films. 2000;373(1):1-5.

2. Karande VS, Mane SH, Pujari VB, Deshmukh LP. (Cd, Mn) Se semimagnetic semiconductor thin films: growth from solution, structural analysis and optical properties. Materials Letters. 2005;59(2-3):148-152.
3. Karande VS, Mane SH, Pujari VB, Deshmukh LP, Wadgaonkar AR. Electrochemical studies of $n-\mathrm{Cd}_{1-x} \mathrm{Mn}_{\mathrm{x}}$ Se thin film photodetector. Journal of Materials Science: Materials in Electronics. 2004;15(7):419-423.

4. Hodes G. Semiconductor and ceramic nanoparticle films deposited by chemical bath deposition. Phys. Chem. Chem. Phys. 2007;9(18):2181-2196.

5. Basu PK, Pramanik P. Solution growth technique for the deposition of cobalt sulphide thin film. Journal of Materials Science Letters. 1986;5(12):1216-1218.

6. Eze FC, Okeke CE.Chemical-bath-deposited cobalt sulphide films: preparation effects Chemical-bath-deposited cobalt sulphide films: preparation effects. Materials Chemistry and Physics. 1997;47(1):31-36.

7. Mane ST, Kamble SS, Deshmukh LP. Cobalt sulphide thin films: Chemical bath deposition, growth and properties. Materials Letters. 2011;65(17-18):2639-2641.

8. Yu Z, Du J, Guo S, Zhang J, Matsumoto Y. CoS thin films prepared with modified chemical bath deposition. Thin Solid Films. 2002;415(1-2):173-176.

9. Okoli DN, Okoli C. Optimal Growth and Characterization of Cobalt Sulphide Thin Films Fabricated Using the Chemical Bath Deposition Technique. Journal of Natural Sciences Research. 2012;2(3):5-8.

10. Zhou J, Bian GQ, Zhu QY, Zhang Y, Li CY, Dai J. Solvothermal crystal growth of $\mathrm{CuSb} Q_{2}(Q=\mathrm{S}, \mathrm{Se})$ and the correlation between macroscopic morphology and microscopic structure. Journal of Solid State Chemistry. 2009;182(2):259-264.

11. Kassim A, Ho SM, Abdullah AH, Nagalingam S. XRD, AFM and UV-Vis Optical Studies of PbSe Thin Films Produced by Chemical Bath Deposition Method. Transactions C: Chemistry and Chemical Engineering. 2010;17(2):139-143.

12. Suresh S, Arunseshan C. Dielectric Properties of Cadmium Selenide (CdSe) Nanoparticles synthesized by solvothermal method. Applied Nanoscience. 2014;4(2):179-184.

13. Suresh S. Studies on the dielectric properties of CdS nanoparticles. Applied Nanoscience. 2014;4(3):325-329.

14. Sagadevan S, Sundaram AS. Dielectric properties of lead sulphide thin films for solar cell applications. Chalcogenide Letters. 2014;11(3):159-165.

15. Suresh S. Synthesis, structural and dielectric properties of zinc sulfide nanoparticles. International Journal of Physical Science. 2013;8(21):1121-1127.

16. Sagadevan S, Podder J. Optical and Electrical Properties of Nanocrystalline $\mathrm{SnO}_{2}$ Thin Films Synthesized by Chemical Bath Deposition Method. Soft Nanoscience Letters. 2015;5(4):55-64.

17. Thirumavalavan S, Mani K, Suresh S. Investigation on structural, optical, morphological and electrical properties of lead sulphide $(\mathrm{PbS})$ thin films. Journal of Ovonic Research. 2015;11(3):123-130. 